\title{
Géo-graphie du Grand Nord. Sur Archipel et Nord
}

\section{Michel Collot}

\section{OpenEdition}

\section{Journals}

Édition électronique

URL : http://journals.openedition.org/ccs/820

DOI : $10.4000 /$ ccs. 820

ISSN : 2558-782X

\section{Éditeur :}

Presses universitaires de Rennes, Association des lecteurs de Claude Simon

\section{Édition imprimée}

Date de publication : 31 décembre 2011

Pagination : $95-112$

ISBN : 9782354121464

ISSN : $1774-9425$

\section{Référence électronique}

Michel Collot, « Géo-graphie du Grand Nord. Sur Archipel et Nord », Cahiers Claude Simon [En ligne], 7 | 2011, mis en ligne le 21 septembre 2017, consulté le 01 mai 2019. URL : http:// journals.openedition.org/ccs/820 ; DOI : 10.4000/ccs.820 


\title{
Géo-graphie du Grand Nord
}

\author{
Sur Archipel et Nord
}

Michel COLLOT

Université Sorbonne nouvelle Paris 3

Institut Universitaire de France

L'œuvre de Claude Simon est fortement liée aux lieux, à la géographie autant qu'à l'histoire. Son histoire est une géohistoire, qui, comme celle de Fernand Braudel, valorise la longue durée, au point d'apparaître comme une histoire immobile ou du moins répétitive. On se rappelle l'épigraphe de L'Invitation, empruntée à Bismarck : "Le seul facteur permanent de l'Histoire, c'est la géographie ». Et réciproquement sa géographie est faite de lieux de mémoire, d'une mémoire collective et personnelle : Les Géorgiques, qui disent l'attachement de Simon à la terre, relèvent du roman historique.

Dans les romans, cette attention aux lieux se traduit par l'expansion de la description qui tend, si ce n'est à " enliser le récit", selon la formule de Jean Ricardou ${ }^{1}$, du moins à l'égarer ; mais elle reste prise dans une logique narrative, si ce n'est fictionnelle. Dans Archipel et dans Nord, deux textes récemment redécouverts, la fic-

1. Jean Ricardou, Le Nouveau Roman, Paris, éditions du Seuil, 1973 p. 128. 
tion disparaît quasiment au profit du documentaire. Si récit il y a, notamment dans Nord, il s'agit d'une relation de voyage, voire d'un reportage. La trame narrative s'y réduit à un parcours de l'espace et laisse le premier plan à la description ou à l'évocation des lieux et des paysages.

Cette prépondérance de la thématique spatiale répond certes à la demande de la rédaction des magazines qui ont publié ces textes brefs $^{2}$; elle me semble pourtant liée aussi à l'accentuation d'une dimension poétique, qu'on trouve ailleurs chez Simon, mais qui s'accuse très nettement ici, notamment dans Archipel, où elle se traduit, entre autres, par une mise en page du texte qui est une mise en espace. Mon hypothèse est que cette poéticité, sous-jacente à toute l'œuvre de Claude Simon, s'affirme ici parce que la description s'y émancipe du cadre de la narration et de la fiction.

Cette promotion de « la forme spatiale " est un phénomène majeur dans l'évolution de la littérature au XX $X^{\mathrm{e}}$ siècle, repéré dès 1945 par le critique américain Joseph Frank dans un article célèbre ${ }^{3}$. Elle concerne non seulement la poésie, qui explore de plus en plus librement l'espace de la page, mais aussi le récit contemporain, qui tend à devenir un "récit d'espace " ${ }^{4}$, et par là-même souvent un " récit poétique " comme le montre, sous des formes et à des titres divers, l'évolution de la production d'un Butor ou d'un Gracq.

Cette évolution a suscité et justifie l'émergence de nouvelles approches du texte, qu'on a pu appeler géographie littéraire, géocritique ou géopoétique et qui sont aujourd'hui en plein essor ${ }^{6}$.

2. Archipel a d'abord paru au Danemark en anglais, suédois, finnois et allemand dans Aland 74, Anders Nyborg, Rungsted Kyst, octobre 1973, p. 19-24 ; Nord, accompagné de traductions en anglais et en allemand, chez le même éditeur, dans Finland 74, décembre 1973, p. 51-65. Les deux textes ont été publiés ensemble en français dans Archipel et Nord (éditions de Minuit, 2009). C'est à cette édition que renvoie la pagination mentionnée entre parenthèses à la suite des extraits de ces textes que je serai amené à citer.

3. Joseph Frank, "La forme spatiale dans la littérature moderne " (1945), Poétique, $\mathrm{n}^{\circ} 10,1976$, p. 244-266.

4. J'emprunte l'expression à Michel de Certeau ("Récits d'espace " dans L'Invention du quotidien, Gallimard, "Folio Essais », tome I, 1990).

5. Voir Jean-Yves Tadié, Le Récit poétique, PUF, 1978.

6. Voir notamment Bertrand Westphal, La Géocritique, Réel, fiction, espace, Mi- 
C'est en m'inspirant de ces démarches que je proposerai trois séries de remarques sur ces textes brefs, encore mal connus mais riches en enseignements pour qui s'intéresse aux croisements entre littérature et géographie.

\section{Géographie}

Ces deux textes ont été publiés en 1973 au Danemark, au moment même de ce qu'on a pu considérer comme le tournant " textualiste " de la production simonienne. Telle est du moins la lecture qu'a tenté d'imposer en 1974 le colloque de Cerisy, dirigé par Jean Ricardou. Or ils me semblent échapper à une telle lecture et à la conception de la littérature qui la sous-tend et que Simon n'a jamais faite sienne, même s'il a pu paraître dans les années 1970 lui donner si ce n'est des gages du moins des arguments. Leur écriture est en effet tournée davantage vers la réalité extérieure que vers elle-même, et elle fait la part belle aux référents, ce qui n'était pas très bien vu alors des avant-gardes littéraires françaises. Dans le texte qu'il a écrit pour accompagner la publication de ses photographies, Simon rappelle ce débat dans lequel il a été pris à partie :

En littérature, les tenants d'une certaine école romanesque ont cru, avec une vigueur et une autorité parfois agressives, pouvoir séparer ce qu'ils ont appelé la dimension référentielle d'un texte de sa dimension « littérale » $(\ldots)$

Au cours d'un colloque (...) il m’a été sévèrement reproché (...) d'avoir fait état, à l'occasion d'une exposition, de mes 'référents', que mon censeur opposait à ce que, dans ce groupe, on appelait les 'générateurs d'un texte ${ }^{7}$.

Cette dimension référentielle tient sans doute à la nature particulière de ces textes, qui sont des récits de voyage, et à leur lieu de publication : les magazines qui les ont accueillis et peut-être com-

nuit, 2007 ; Kenneth White, Le Plateau de l'Albatros. Introduction à la géopoétique, Grasset, 1994 ; et mon article "Pour une géographie littéraire ", dans le numéro 8 de la revue en ligne $L H T$, consultable à l'adresse : http://www.fabula.org/lht/8 7. Claude Simon, Photographies: 1937-1970, Maeght, 1992, p. 15-16. 
mandés à Simon ne sont pas des revues littéraires mais plutôt des fascicules à vocation touristique. Ils sont issus de notes prises sur place, qui ont pu être " complétées » et " mises en ordre » après-coup comme celles d'un voyage en Zeeland publiées peu de temps avant dans la revue Minuit $^{8}$. Mais celles-ci ont été soumises à un travail de textualisation et fictionnalisation qui annonce très directement Les Géorgiques, comme si, pour publier dans la revue parisienne d'un éditeur d'avant-garde, Simon avait estimé nécessaire d'atténuer la dimension référentielle de ses notes de voyage, alors qu'il s'y adonne plus librement dans les textes parus presque à la même date dans deux revues danoises inconnues de ses pairs, et, pour l'un d'entre eux, dans d'autres langues que le français.

On pourrait en conclure que ces textes, publiés dans des lieux excentriques, occupent une place très marginale dans l'œuvre de Claude Simon. Il n'en est rien à mes yeux : ces deux petits textes, si tardivement publiés en France, me semblent avoir été plutôt en avance sur la suite de la production simonienne et sur l'évolution générale des pratiques littéraires, qui vont l'une et l'autre, à partir des années 1980, donner au récit de voyage et à l'autobiographie une importance croissante. Comme quoi les lieux de publication marginaux sont parfois plus favorables à l'innovation que ceux qui sont situés au centre, plus soumis à l'air du temps.

Archipel et Nord se présentent un peu comme des "reportages ", ancrés dans la réalité des contrées visitées par l'écrivain-voyageur. Il en décrit très précisément la géographie, la topographie, la végétation, la géologie, l'habitat, sans oublier d'en rappeler l'histoire. Simon semble ici se référer principalement à la connaissance directe qu'il a pu prendre des lieux qu'il évoque, même s'il a sans doute fait appel à une documentation complémentaire, qui a pu lui être fournie par ses hôtes. Plus que sur des choses lues, il s'appuie sur des choses vues et entendues, objets d'une expérience sensible et vécue.

L'archipel qui donne son titre au premier texte est celui d'Åland, constitué de six mille cinq cents îles et îlots situés entre la Finlande et la Suède. On peut suivre sur une carte le trajet que le visiteur a ef-

8. "Essai de mise en ordre de notes prises au cours d'un voyage en Zeeland (1962) et complétées ", Minuit, n³, mars 1973, p. 1-18. 
fectué pour le rejoindre en avion depuis Helsinki, survolant d'abord une côte très découpée, à l'intérieur de laquelle l'eau s'insinue par de multiples échancrures, puis une mer parsemée de " débris " de terre. L'écrivain s'est intéressé à la géologie de cet archipel, constitué de granit rose érodé par les glaciations du Pléistocène ; il en évoque poétiquement la lente formation :

millions d'années aux épaisseurs bleuâtres rampant rabotant dans un formidable silence peuplé de formidables craquements le granit poli milliers d'îles milliers de golfes de baies de criques où s'arrondit la mer couleur d'huître (p. 11).

Ce travail de l'érosion glaciaire explique à la fois la disposition des îles " en longs chapelets parallèles » et leur relief caractéristique, le granit ayant été tellement aplani que certains îlots, "pas assez hauts pour qu'une seule vague ne les recouvre", ressemblent à des " poisson(s) pétrifié(s) " (p. 15) : "l'eau s'étalant en nappe sans se briser sur la surface polie du granit se retirant la laissant mouillée flanc d'une baleine lilas » (p. 13). En bon observateur, Simon, guidé par ses accompagnateurs, remarque les stries laissées par le glacier au cours de son déplacement : " non pas roc mais cuir épais de vieux pachyderme sillonné de rides de fissures d'entailles entrecroisées laissées par un couteau ébréché » (p. 13). À son regard informé n’échappent pas non plus les lichens qui recouvrent beaucoup de rochers, " gris-vert ou jaunes comme des pièces de monnaies mordant les unes sur les autres » (p. 15), ni les végétaux caractéristiques de la taïga : " touffes d'aulnes sorbiers frissonnant à peine et ces longues herbes comme des plumes roses formant de loin des nuages estompés pastel »(p. 12-13).

Les notations concernant l'agriculture et l'habitat de l'archipel sont plus fugitives, et je n'y insisterai pas ${ }^{9}$. Les allusions historiques sont en revanche nombreuses et insistantes, mais dispersées ; elles ne s'organisent évidemment pas selon un ordre chronologique, mais interviennent à l'occasion de la visite de quelques lieux de mémoire. Ces derniers ne sont jamais nommés, mais on peut les identifier

9. Voir par exemple l'évocation des " maisons aux toits verts aux parois rouges aux croisées blanches » et des champs qui les entourent, p. 14. 
d'après les monuments qui y sont décrits ou les événements qui s'y sont déroulés . Ainsi lorsque Simon relate une excursion en hydravion jusqu'à l'île de Kökar, il mêle à la description des vestiges du monastère et des fresques qui le décorent l'évocation de l'arrivée des moines-soldats qui l'ont fondé au XIV siècle :

Franciscains moines fanatiques déchaux venus d'où construire ici un sanctuaire de blocs roses lilas bistre cyclamen au toit couvert d'écailles peindre le flagellé le juge en robe prune qui se lave les mains sculpter ces grappes de sang coagulé $[\ldots]$

casqués et armés de fer eux aussi sans doute ils avaient pris pied sur ces mêmes rochers débarquant de nacelles cloutées ceints de baudriers par-dessus leurs robes brunes (p. 12-13)

Plus loin, c'est la visite de la forteresse de Bomarsund qui rappelle le siège qu'Anglais et Français lui ont fait subir pendant la guerre de Crimée, immortalisé par une "gravure " (p. 15-16) minutieusement décrite par Simon. Les conflits qui ont opposé pendant des siècles les «princesses » danoises et russes pour la possession de ces îles sont évoqués plus discrètement vers la fin du texte (p. 16), mais celui-ci se termine sur une extraordinaire énumération des différentes composantes de la topographie de l'archipel (" détroits ", « isthmes " " îles », "lacs ", " marécages ", " criques ") avant de se résoudre dans "le blanc du silence » (p. 17). La géographie l'emporte ici sur l'histoire, et la paix de la nature sur les violences des hommes : tout au long du texte, Simon oppose implicitement aux " fleurs » et au " tonnerre » " de feu " déchaînés par les envahisseurs la végétation paisible et le silence qui règnent désormais sur ce sanctuaire naturel :

nénuphars cernés de clair s'éparpillant sur le fond d'ardoise dérivant $[\ldots]$

tonnerre soudain dans ces silences fleur de feu au cœur jaune aux pétales vermillon s'épanouissant combats pour ces détroits [...]

fleurs tonnantes de feu étraves bardées de fer [...] comme de sacrilèges coups de tonnerre se répondant dans le silence répercutés par les glaces les roches le ciel vide (p. 11 et p. 15). 
Au terme d'une succession interminable de guerres, l'archipel d'Åland a été démilitarisé en 1922 ; bien que rattaché à la Finlande, il est devenu autonome et un traité garantit sa neutralité. Il est aujourd'hui à l'image de la Scandinavie telle que la voit Simon : « une sorte d'îlot privilégié et exemplaire " " en bordure du monde de fer et de violence où nous vivons $»^{10}$.

La composition de Nord est encore plus nettement géographique. Ce texte est construit sur une opposition entre le sud de la Finlande, civilisé et quasi méridional («sudiste»), et « le Grand Nord, superbe et sauvage " (p. 27). La trame narrative, beaucoup plus continue, suit très précisément le trajet effectué de la capitale du pays à son extrémité septentrionale et retour. Le narrateur et le récit partent du port d'Helsinki, avec ses "façades à frontons et à colonnades " " peintes de délicates couleurs pastel » (p. 21), traversent la ville, avec ses parcs et ses statues (p. 22-23), gagnent la campagne puis des zones de plus en plus sauvages, où les cultures disparaissent au profit de la forêt (p. 24), d'abord cultivée puis inculte, décimée par les tempêtes ; la route fait alors place à une " piste sablonneuse " (p. 25).

Cet itinéraire aboutit à Sevettijärvi, village situé à l'extrême nord du pays, en Laponie finlandaise, bien au-delà du cercle polaire. Les Lapons skolts, chassés de leur territoire par les Russes en 1945, y ont été établis et conservent vivantes leurs coutumes, comme le tri hivernal des rennes, évoqué par le narrateur parmi d'autres usages (p. 31). Le récit de ce voyage, que Simon a pu faire en voiture l'été ${ }^{11}$ (puisqu'il évoque le soleil de minuit, la nuit blanche) est prolongé par la mention d'un autre trajet le long du cercle polaire, qui parcourt d'ouest en est la Sibérie, de Kandalachka à la mer des Tchoukches et qui mène "plus loin qu'un homme ne pourrait aller en marchant toute une vie " (p. 25). Simon a pu l'effectuer en avion lorsqu'il est allé au Japon ; son évocation nous fait sortir des frontières de la Finlande et étend le territoire du Nord à sa dimension continentale voire planétaire, selon un mouvement d'ouest en est dont j'interrogerai plus loin les résonances.

10. Discours de Stockholm, dans Euvres, éd. A. Duncan, Gallimard, "Bibliothèque de la Pléiade ", 2006, p. 288.

11. En août 1973, d'après Mireille Calle-Gruber (voir Claude Simon. Une vie à écrire, Seuil, 2011, p. 305). 
Le voyage en Laponie est suivi d'un retour vers le sud et vers la civilisation occidentale (p. 32) et le récit se termine où il avait commencé : dans le port d'Helsinki. Orienté vers le Nord, mais revenant à son point de départ méridional, le trajet du narrateur a un sens, qui n'est pas seulement géographique, mais aussi symbolique. Une géographie littéraire ne peut pas se borner à identifier les référents géographiques d'un texte, elle doit étudier la façon dont il leur donne sens et forme. C'est la tâche d'une géocritique et d'une géopoétique.

\section{Géocritique}

La géocritique, telle que l'entend Bertrand Westphal, est une méthode essentiellement comparatiste, qui consiste à confronter les différentes images que donnent d'un lieu divers écrivains. C'est une démarche intéressante, mais je ne l'adopterai pas ici. Plutôt que de comparer Archipel et Nord aux textes d'autres écrivains consacrés aux mêmes contrées, je préfere les mettre en rapport avec d'autres œuvres de Simon lui-même. Je laisserai donc un peu de côté l'intertextualité externe, qui joue un rôle dans ces textes, à travers de multiples allusions (notamment à Tchekhov, à Rimbaud, à Hegel, aux légendes scandinaves) et privilégierai l'intertextualité interne, afin de cerner plus précisément la spécificité de la vision simonienne de ces lieux.

Tel est, selon moi, l'objet propre de la géocritique, qui la distingue d'une géographie référentielle : étudier non plus le pays mais l'image du pays, c'est à dire le paysage, et non plus la carte. Cette notion que j'entends dans un sens proche de celui que lui a donné Jean-Pierre Richard ${ }^{12}$, me semble d'autant plus pertinente ici que ces textes, comme beaucoup d'autres de Simon, mettent l'accent sur le rôle de la perception en général, et du point de vue en particulier, dans l'élaboration de cette image. Il y a par exemple une sorte de réalisme phénoménologique dans l'évocation initiale de l'archipel vu d'avion, où le narrateur se montre attentif aux « effets

12. Voir à ce propos ma mise au point dans Paysage et poésie, Corti, 2005, p. 177-189. 
d'optique " (p. 9). La précision de ce regard de photographe n'exclut d'ailleurs ni le recours aux autres sens, à l'ouïe notamment, très sensible, on l'a noté, au silence des lieux, ni l'intervention de l'imaginaire : en même temps qu'il découvre ces îles, le voyageur « les imagin(e) peuplées de créatures fabuleuses » (p. 11)

Tout cela donne une forme et une signification bien particulières à ces deux archétypes géographiques que sont l'archipel et le Grand Nord. Ils ont en commun le dépaysement qu'ils procurent au voyageur, au sens le plus fort du terme : un bouleversement de sa vision du monde. Ce sont des terres inconnues, en marge de la civilisation : on pourrait interpréter en ce sens les toponymes Aland et Finlande, qui désigneraient des bouts du monde, voire une fin du monde. Nous avons vu que l'âpreté du Grand Nord s'oppose à la civilisation trop raffinée du sud. On sait l'attrait qu'exerce sur Simon cette sauvagerie qui délivre les lieux de l'emprise de la culture, voire de l'homme, et qui permet d'accéder à un réel dépouillé des représentations qui l'occultent habituellement.

Au début d'Archipel, ce changement de point de vue est favorisé notamment par la perspective aérienne que permet le survol en avion et que Simon adopte souvent, parce qu'elle minimise les traces de la civilisation et de la présence humaine. Elle brouille ici la distinction familière et fondamentale entre le ciel et la mer, le haut et le bas : tout se passe " comme si au-dessous du tissu de prés de bois de champs parallèles s'étendait un autre ciel, symétrique à celui où vole l'avion" (p. 9). Et au moment de l'atterrissage, "la mer l'archipel tout entier » semblent « mont(er) vers les passagers de l'avion" (p. 12).

La topographie des côtes finlandaises et de l'archipel favorise en outre l'indistinction entre la terre et l'eau ; les terres sont trouées de lacs, et la mer, parsemée d'îles, si bien que les rapports entre figure et fond, creux et pleins, sont perturbés voire inversés : miroitant dans la lumière, les étendues liquides qui s'infiltrent entre les terres apparaissent non pas comme des " trous ", mais comme des "flaques de mercure répandu faiblement en relief sur la terre assombrie » (p. 9). Toute idée de continuité continentale et territoriale vole en éclats : la terre "se déchiqu(ète) se dépiaut(e) pour ainsi dire / haillon percé 
de mille déchirures" (p. 10). Et l'altitude fait perdre l'échelle des choses : la mer devient une "mare » (p. 10) et la campagne, miniaturisée, un " jardin » japonais (p. 14).

Cette désorientation générale produit l'image d'un monde à l'envers. Le motif de l'inversion, familier aux lecteurs de Simon, revient comme un leitmotiv dans l'évocation de l'archipel :

la route obliquant vers le haut s'incurvant revenant sur la gauche en suivant la rive s'incurvant en sens inverse [...]

l'inverse à présent : lambeaux s'étirant en longs chapelets parallèles

Ce renversement culmine dans l'évocation de l'archipel des Cyclades qui se superpose à celui d'Åland, et du drapeau de la Grèce, image inversée de celui de la Finlande :

archipel АРХI-ПЕ $А$ АГО $\Sigma$ : primitivement non ces innombrables grains de terre semés mais au contraire la vaste mer

comme si le sens s'était inversé Contenant pour le contenu Grèce à l'envers (et de même les deux drapeaux l'un à croix blanche sur fond bleu l'autre à croix bleue sur fond blanc) Comme un positif photographique et son négatif sablier le haut en bas où le vide est plein langage retourné comme un gant les coutures ici devenant saillies (p. 11)

Aux illusions d'optique s'ajoutent les équivoques sémantiques et symboliques. Le retour à un sens supposé premier du mot archipel, qui signifiait en grec ancien "la mer principale ", équivaut à une sorte de submersion linguistique des îles, tandis que le bleu et le blanc échangent leurs positions respectives dans les pavillons de la Grèce et de la Finlande. Si la littérature reflète le réel, c'est un reflet inversé comme sur un négatif photographique ou dans un miroir. En changeant le sens des mots, elle révèle l'envers de la réalité familière, que nous cachent nos habitudes de pensée et de langage. Ce motif du monde à l'envers est très présent dans l'œuvre de Simon ; par exemple dans ce passage des Géorgiques, qui reprend la même image du gant retourné : «le monde à présent pour ainsi dire retourné à la façon d'un gant, d'un vêtement, révélé dans son envers ou plutôt 
perverti en ce sens que plus rien n'y avait la même signification, sinon de signification tout court $»^{13}$.

Si un tel renversement est une épreuve, c'est aussi un révélateur. En inversant notre point de vue, la littérature nous fait accéder à une vision plus lucide des choses. L'image chaotique et fragmentée qui nous est donnée de l'archipel déjoue les canons classiques de la description, mais elle correspond à une autre organisation du texte, fractale et fragmentaire. Du chaos procède un nouveau cosmos, peut-être un chaosmos, dont "les triangles de glace " qui s'entrechoquent dans le port d'Helsinki, " chaos de plaques grisâtres en étoiles " offrent l'image, inquiétante et fascinante (p. 22).

Le Grand Nord est lui aussi un monde à l'envers : en juin, il y fait jour à minuit (p. 22); la nuit est laiteuse et blanche (p. 32). On rencontre "en pleine campagne " des couples " en costumes de ville " qui paraissent "irréels " comme les " messieurs de bronze " assis dans les squares (p. 23) ou " le cheval un instant entrevu " en chemin :

immobile comme suspendu dans l'air comme dans un film au ralenti longeant la route derrière la clôture du pré irréel sans couleur lui non plus animal sorti de quelque légende nocturne dans le demi-jour participant de cette même irréalité que la nuit elle-même (p. 23-24).

La distinction entre le rêve et la réalité, le réel et sa représentation, vacille, et, avec elle, le règne de la raison : là où la nuit ne tombe jamais, «l'oiseau de Minerve » cher à Hegel ne saurait prendre son envol (p. 33). Irréel et irrationnel, cet univers est foncièrement ambivalent, féérique comme un " conte nordique » (p. 32) et inquiétant, tels « les troncs minces argentés et roses " des bouleaux, "parsemés de taches noires " qui semblent autant de "blessures" (p. 28). Ces motifs sont repris, parfois littéralement, dans des textes qui évoquent l'hiver 39-40 ${ }^{14}$. Mais ils prennent un sens différent, dans un contexte tout autre : celui de la guerre, et d' " un paysage enneigé ", figé par un froid glacial ; dans Les Géorgiques, le nom d'Anor est associé au

13. Les Géorgiques, Minuit, 1981, p. 426.

14. Voir "Progression dans un paysage enneigé "(Études littéraires, IX, $\mathrm{n}^{\circ} 1$, 1976, p. 21-23) et Les Géorgiques. 
noir et à la mort, alors qu'ici, la blancheur du Grand Nord est liée à la clarté des nuits d'été et à la rapidité des "galopants coursiers d'écume ", semblables à " de la neige » (p. 27).

Même les évocations du terrible hiver polaire semblent plutôt trahir la fascination du narrateur. Bien que sauvage et rude, loin d'être inhumain et désert, ce paysage est plein de vie : le voyageur $\mathrm{y}$ rencontre des hommes accueillants et des animaux particulièrement gracieux : renne, écureuil, lièvre, chien. Le Grand Nord semble être le lieu d'un retour aux sources, aux origines du monde et de l'humanité ; c'est peut-être la raison pour laquelle Simon a greffé sur le récit de ce voyage en Finlande l'évocation d'un trajet qui l'a conduit jusqu'en Extrême-Orient. Le narrateur y " avance » à la fois "dans la vieillesse » (p. 24) et "dans l'enfance du monde» (p. 25); l'alternance entre les deux formules suggère leur équivalence : elles désignent l'archaïque, au double sens du très ancien et du commencement.

La rêverie sur la préhistoire, présente dans Archipel à travers l'évocation des glaciers du Pléistocène, s'inscrit ici dans le motif des cimetières d'arbres, comparés à des insectes géants "d'avant le déluge » (p. 34), et des cimetières d'animaux " gisant là par troupeaux entiers avec leurs cornes enchevêtrées tordues et grises que le gel les hivers fendillent peu à peu " (p. 28-29). Mais ces cimetières ne sont pas seulement des lieux de conservation, comme ceux de L'Invitation où « un formidable phénomène glaciation conserv(e) intacts - sinon comestibles - les corps de gigantesques mammouths " ${ }^{15}$, mais des lieux de régénération : les organismes vivants qui s'y décomposent donnent naissance à d'autres formes de vie, alimentant sans cesse le cycle des morts et des renaissances. C'est le cas notamment des arbres abattus par les tempêtes : ils forment un chaos qui est aussi une " matrice d'arbres ". " naissant, croissant impétueux traversant l'acier des hivers" (p. 26).

"Les cimetières du monde » sont aussi les lieux de sa renaissance. La vision de ces «squelettes emmêlés couchés parmi les vivants avec leurs fantastiques racines " rappelle moins le souvenir des danses macabres du Moyen-Âge que le mythe d'un chaos créateur, ancré dans

15. L'Invitation, Minuit, 1987, p. 74. 
l'imaginaire collectif. Les « marécages gluants » (p. 32) qui ont donné son nom à la Finlande (" fin-land suo-mi : terre des marais", p. 11) inscrivent dans son paysage ce mélange fécond des éléments qui préside à la naissance de la vie. Monter vers le Nord, c'est remonter le temps. Les habitants y ont gardé des usages et un mode de vie antérieurs à l'irruption de la technique et des mœurs urbaines (p. 30); et le " très vieil homme " auquel s'identifie le voyageur ( « je demandai son âge on me le dit c'était le mien ", p. 29-30) incarne ce passé immémorial où l'humanité vivait en accord avec la nature. Loin de la civilisation chrétienne qui nous incite à dépouiller le vieil homme, le Grand Nord semble nous inviter à renouer avec le paganisme.

Mais le voyageur sait que c'est utopique, et il renonce à rencontrer «le très vieil homme de la rivière " (p. 32), pensant qu' il a "droit au silence à la paix là-bas au bord du vieux grand fleuve » (p. 33). Il doit se résigner à s'éloigner du Nord sauvage et à rejoindre la civilisation, ce qui est aussi une manière de revenir au commencement du voyage et du récit.

Celui-ci se termine sur une autre évocation de la jeunesse du monde : celle des adolescents assis sur le quai, qui ressemblent à tous ceux qui peuplent les villes occidentales. Bien qu'ils soient les produits d'une civilisation décadente, le narrateur semble jeter sur eux un regard plutôt bienveillant. Même s'il est de l'âge du très vieil homme de la rivière, et si le vent du nord finalement l'emporte, il parât s'être un moment réchauffé au contact de leur jeunesse. Et c'est à eux que reviennent presque les derniers mots : ceux de la chanson finlandaise qu'ils se mettent à chanter en s'accompagnant de leur guitare. Ses sonorités séduisent d'autant plus le voyageur que leur sens lui échappe (p. 34-35), ce qui est le propre des chansons et des langues étrangères, mais peut-être aussi de la poésie.

\section{Géopoétique}

Le sens de cette géographie revisitée par l'imaginaire simonien est indissociable de la forme qu'elle revêt dans ces textes et qu'on peut qualifier de poétique. Cette dimension poétique est présente aussi dans les textes romanesques de Simon, mais elle est ici particulièrement saillante du fait de son émancipation vis à vis de tout cadre 
narratif et fictionnel. C'est surtout vrai dans Archipel, la trame narrative étant plus marquée dans Nord, comme l'indiquent l'usage du passé simple et la présence de nombreux marqueurs de temps, qui scandent les différentes étapes du voyage. Ce récit n’en comporte pas moins lui aussi des traits de poéticité, communs à l'onomastique, à la typographie, à la syntaxe, et à la rhétorique de ces deux textes brefs.

La mention récurrente de toponymes étrangers permet la mise en valeur de leurs signifiants, puisqu'ils n'ont pas de signifiés, et que leur référent n'est pas nécessairement connu du lecteur. C'est particulièrement net dans l'énumération des noms de lieux sibériens dont les sonorités rudes évoquent la sauvagerie d'un monde étranger à l'homme :

Ainsi jusqu’à Kandalachka et après Kandalachka Arkhangelsk et après Arkhangelsk Vorkouta et après Vorkouta Igarka et après Igarka Salekhad les plaines les plateaux les montagnes de Verkhoiansk celles de Tcherskii ainsi jusqu'à l'Anadyrle Baïkal la mer des Tchoukches (p. 25).

On sait la "fascination" de Simon "pour les idiomes incompréhensibles aux consonances étranges " ${ }^{16}$. À la fin de Nord, il se dit sensible à la musicalité rauque du finnois, cette "langue gutturale à la fois violente et tendre ", et il transcrit sans les traduire les paroles d'une chanson, leur rythme et leurs sonorités étant porteurs d'une expressivité qui ne relève pas de la signification mais de la signifiance : "j’imagine que comme dans toutes les chansons le sens des mots n'a pas d'importance Seulement leurs sons leur musique » (p. 35) ${ }^{17}$. Il est également attentif à la dimension graphique des mots finlandais, qui comportent " des voyelles des consonnes redoublées s'étirant suspendues comme à des poteaux à la hampe des lettres dures les $\mathrm{T}$ les $\mathrm{K}$ semblables à des étais des cassures " (p. 34). L'inscription en capitales du nom de la capitale finlandaise donne un relief particulier aux lettres qui le composent; en les isolant, le narrateur les donne à voir plutôt qu'à lire, insistant sur leur forme, qui

16. Le Jardin des plantes, Minuit, 1997, p. 65.

17. Le sens des paroles citées par Simon est, de fait, bien décevant, très éloigné de la rêverie qu'elles lui inspirent : "Comme il me plairait comme il me plairait / de parler d'un grand bonheur / des nectars de la terre et du ciel / et de l'amour ». 
entre en relation mimétique avec leur support (les drapeaux) et avec le paysage du port pris dans les glaces :

HELSINGFORS bleu rouge jaune terminé par un drapeau F flottant dans le vent ondulant S, HELSINKI se brisant sur le K comme ces triangles de glace fracassée par les étraves (p. 21).

La transcription en lettres grecques capitales du mot APXIПЕ $\triangle А Г O \Sigma$ (p. 11) redouble l'effet d'étrangeté et de dépaysement par l'emprunt à un autre alphabet qui rend pour certains lecteurs au moins l'inscription non seulement illisible mais imprononçable. C'est un procédé fréquent dans l'œuvre de Simon, qu'on trouve en particulier dans La Bataille de Pharsale, et qui met en valeur la dimension graphique, voire plastique des mots. L'écrivain se fait peintre non seulement quand il décrit les paysages mais quand il évoque les impératrices aux «noms bleus et blancs bleus et rouges Alexandra Kristina Katherina » (p. 16).

La mise en page de ces textes les soumet à un travail de fragmentation et de spatialisation comparable à celui qu'on rencontre dans la poésie contemporaine, et en tout cas beaucoup plus poussé que dans les autres œuvres de Simon, où la continuité typographique masque le plus souvent les discontinuités sémantiques et syntaxiques. Les blancs et les alinéas viennent remplacer ici la ponctuation, presque totalement absente à la fin des séquences. Ils isolent notamment de façon spectaculaire certains syntagmes, qui, dès lors, se rapprochent visuellement, et parfois prosodiquement, du vers. Leur poéticité est d'autant plus marquée qu'il s'agit souvent de métaphores, et elle est renforcée par des recherches de rythme et de sonorité : « haillon percé de mille déchirures " (p. 10, décasyllabe) ; " clapotis de silence " (p. 14, hexasyllabe, allitération et assonance).

Beaucoup d'énoncés plus longs s'interrompent eux aussi avant la justification, sans attendre que la phrase et le sens soient complets, s'arrêtant parfois net sur un mot outil qui appelle une suite qui ne viendra pas, imitant l'effet produit en poésie par le rejet ou le contre-rejet :

colonnes processions de pèlerins cheminant fantastique armada vers (p. 10). 
Les paragraphes s'apparentent à des strophes, ponctuées parfois d'anaphores ou de leitmotive, telle la répétition obsédante du mot " silence " dans Archipel, créant des parallélismes analogues à ceux de la poésie, particulièrement apparents dans la longue énumération qui conclut le texte: "les détroits // les isthmes // les îles nénuphars // les lacs de mercure // les processions d'îles // les marécages // les caravanes d'îles sur la mer d'étain // les criques // les joncs pâles » (p. 17). Cette série de brefs syntagmes alignés verticalement sur la page crée un rythme visuel inhabituel, où les blancs l'emportent sur les noirs, donnant à voir le rapport de l'écriture avec « le blanc du silence " qui est essentiel à la poésie. On a ici affaire à un véritable archipel typographique, l'espacement du texte correspondant à la dispersion des îles à la surface de la mer, nous donnant à lire cette " parole en archipel " qui est, selon Char, celle de la poésie.

Cette discontinuité typographique se double, dans Archipel, d'un véritable éclatement de la syntaxe. À la différence de Nord, ce texte ne comporte aucune phrase complète, du fait de l'usage systématique de la tournure nominale. Celle-ci est caractéristique du style de l'annotation. Tout se passe comme si Claude Simon avait délibérément renoncé à mettre ses notes en phrases et en phase les unes avec les autres. Mais la syntaxe nominale est aussi très présente dans la poésie contemporaine, qui cultive volontiers « le style substantif " ${ }^{18}$, dressant le mot dans son superbe isolement comme " un signe debout ${ }^{19}$. Dans les autres textes de Simon, elle est le plus souvent en général réservée aux passages descriptifs, comme dans cette belle évocation du soleil de minuit :

une autre fois le soleil orange encore pâlissant peu à peu devenant rose dans le ciel rose de minuit où s'étiraient de longues traînées rousses retroussées comme laissées par les soies d'un pinceau brumes ou fumées des hautes cheminées parallèles brun-rouge les grues des docks enchevêtrées se découpant en noir sur le ciel fardé (p. 22).

18. Souvent pris à partie par Henri Meschonnic, par exemple dans Dédicaces Proverbes, Gallimard, 1972, p. 8.

19. Voir Roland Barthes, "Y a-t-il une écriture poétique ? ", Le Degré zéro de l'écriture [1953], Seuil 1972, collection « Points », p. 39. 
Tout se passe comme si la description était dans Archipel généralisée, au point que la distinction et la hiérarchie entre narration et description semblent effacées ou renversées ; en quoi ce texte apparemment marginal est peut-être la réalisation la plus radicale d'un idéal auquel Simon a souvent rêvé : " n’est-il pas possible de concevoir un système romanesque entièrement neuf, entièrement inversé, c'est-à-dire où la description deviendrait tout de bon l'élément principal ${ }^{20}$. Or on a pu montrer les affinités entre poésie et description, qui l'une et l'autre explorent davantage l'axe paradigmatique que l'axe syntagmatique du discours ${ }^{21}$.

La syntaxe nominale, c'est aussi le style de la "chose vue " : un énoncé tourné vers son objet qui fait l'économie du sujet de l'énonciation $^{22}$. Et de fait, on ne trouve dans Archipel aucune occurrence du pronom de la première personne. S'agirait-il d'un exemple de "poésie objective ", conforme au vœu de Rimbaud ? L'absence de prédication me semble pouvoir être interprétée plutôt comme l'expression d'une expérience antéprédicative, antérieure au jugement, où le sujet ne se distingue pas du monde ${ }^{23}$. Elle serait ici au service de cette écriture de la sensation que cultivent aussi bien Claude Simon que la poésie contemporaine ${ }^{24}$.

Le " principe d'équivalence " qui, selon Jakobson, domine en poésie, régit fortement les textes de Simon, aussi bien dans leur composition (effets d'échos, de répétition, de correspondance) que dans le détail de l'écriture. La présence de figures d'analogie dans Archipel et de Nord n'a donc rien pour surprendre le lecteur, habitué en particulier à l'usage récurrent de la locution " comme si ». Mais les

20. "Roman, description et action ", in Paul Hallberg (dir.), The Feeling for Nature and the Landscape of Man, Göteborg, Rundquists Boktryckeri, 1980, p. 87.

21. Voir en particulier les remarques de Philippe Hamon sur « le poétique et le descriptif " dans "L'œuvre poétique ", Le Grand atlas des littératures, Encyclopaedia universalis, 1990, p. 44-45.

22. Voir Émile Benvéniste, "La phrase nominale ", dans Problèmes de linguistique générale, Gallimard, 1966, p. 159.

23. Voir mon approche de "La syntaxe nominale " dans La Matière-émotion, PUF, 1997, p. 282-285.

24. Voir David Zemmour, Une syntaxe du sensible: Claude Simon et la perception, Presses de l'Université de Paris-Sorbonne, 2008 ; et Nicolas Castin, Sens et sensible dans la poésie moderne, PUF, 1998. 
métaphores in absentia sont ici particulièrement fréquentes, et ce qui accentue leur effet poétique, c'est leur isolement syntaxique et typographique, qui distend les liens entre le comparé et le comparant :

nénuphars cernés de clair s'éparpillant sur le fond d'ardoise dérivant (p. 11).

Séparé par le blanc de tout contexte immédiat, cet énoncé peut se lire de multiples façons. S'agit-il d'une métaphore désignant les îles dispersées à la surface de la mer, qui seront plus loin qualifiées d' "îles nénuphars " (p. 17) ? Ou s'agit-il d'un énoncé littéral désignant de vrais nénuphars, puisque le narrateur signale la présence, parmi la végétation des marais, d'une "fleur de feu au cœur jaune aux pétales vermillon " ? À moins que cette "fleur de feu " soit elle-même métaphorique, comme celles qui désignent la flamme des fusils et des canons (p. 15) ? Toutes ces significations sont possibles et se superposent plutôt qu'elles ne s'excluent, et une telle polysémie contribue elle aussi à donner à ces textes un caractère poétique.

La géographie du Grand Nord est une source d'inspiration pour Simon, non seulement parce qu'elle le dépayse et bouleverse sa vision du monde, mais parce qu'elle lui suggère une forme d'écriture nouvelle, qui se rapproche plus nettement de la poésie, notamment par le rôle qu'elle accorde à la spatialisation du texte. Celle-ci confirme l'importance que revêt l'espace dans la poétique de Simon : on sait qu'il aime évoquer le travail de l'écriture à l'aide de métaphores spatiales, comme les célèbres " mots-carrefours ", et qu'il lui arrive de visualiser la composition de ses textes par des schémas, parfois directement inspirés d'un modèle géologique ou géographique : ainsi du croquis résumant, dans "La fiction mot à mot ", la structure de La Route des Flandres, "l'ensemble se présentant en somme un peu comme ces coupes de terrain au centre desquels se trouve un puits artésien $»^{25}$. De cette géo-graphie simonienne, Archipel et Nord sont deux expressions radicales, et nullement marginales.

25. "La fiction mot à mot ", Euvres, op. cit., p. 1199. 